\title{
Proteomic pattern of cervico-vaginal fluid (CVF) in an ovarian cancer diagnosis - pilot study
}

\author{
Emilia Gasiorowska1', Bartosz Urbaniak², Jakub Lorek¹, Jan Matysiak², Ewa Nowak-Markwitz¹ \\ ${ }^{1}$ Department of Gynecology, Obstetrics and Gynecologic Oncology, Division of Gynecologic Oncology, \\ Poznan University of Medical Sciences, Poznan, Polska \\ ${ }^{2}$ Department of Inorganic and Analytical Chemistry, Faculty of Pharmacy, Poznan University of Medical Sciences, Poznan, Polska
}

\begin{abstract}
Objectives: High grade serous ovarian cancer (HGSC) is the most common type of ovarian cancer and is responsible for about $90 \%$ of ovarian cancer deaths. The diagnostic tests currently used do not increase the detection rates for ovarian cancer. There is a great necessity to develop new and non-invasive diagnostic tests for ovarian cancer (OC). Cervico-vaginal fluid (CVF) seems to be a potential and valuable source of biomarkers for genital tract diseases including ovarian cancer. The aim of our pilot study was to undertake a preliminary proteomic analysis of CVF derived from ovarian cancer patients and to compare these with results from a control group.

Material and methods: We analysed and compared samples from a group of ovarian cancer patients and a control group of healthy patients. The study used MALDI-TOF coupled with nanoLC and ClinProTools software for MS, MS/MS spectra collection and proteomic analysis.

Results: We identified 404 different proteins in the OC group and 417 proteins in the control group. 239 of the proteins were found to be common to both study groups, 165 proteins were unique to the $O C$ subjects, and 178 proteins were unique to the control subjects. We selected three proteins as the OC markers with the greatest potential: cysteine-rich secretory protein 3, fibronectin and Ly6/PLAUR domain-containing protein 3.

Conclusions: The proteins we selected seem to possess great potential as markers for the screening and early detection of OC, especially in non-invasive and low-cost diagnostic tests. However, our findings require more advanced and validated proteomic analysis to confirm the suitability of the selected proteins in everyday medical diagnoses.
\end{abstract}

Key words: ovarian cancer; proteomic pattern; tumor markers; cervico-vaginal fluid

Ginekologia Polska 2018; 89, 12: 688-694

\section{INTRODUCTION}

High grade serous ovarian cancer (HGSC) is the most common type of ovarian cancer and is responsible for about $90 \%$ of ovarian cancer deaths [1]. Early detection of cancers, especially of serous ovarian cancer, is necessary to reduce mortality rates. Until now, various diagnostic tests and tumor markers have been used, but they have not increased the detection of the disease. A randomized study of the applicability and efficacy of these tests in postmenopausal women for ovarian cancer screening showed no reduction in mortality $[2,3]$. It is therefore necessary to seek new tumor markers from body secretions other than blood serum. Cervico-vaginal fluid (CVF) is a potential source of biomarkers for genital tract diseases. It is easily available, and so it is possible to use it in repeatable, inexpensive and non-invasive tests. CVF is a complex body secretion consisting of a mixture of plasma proteins, inflammatory cells, enzymes and genital tract epithelial cells. The body's physiological and disease states are reflected in the proteomic profiles characteristic of CVFs [4]. Determining the proteomic profile for ovarian cancer may allow the identification of new markers of the early stages of this disease.

The aim of our pilot study was to undertake a proteomic analysis of cervico-vaginal fluid in patients with ovarian cancer and to compare the results with those derived from a healthy control group. The study attempted to compare the protein patterns of the ovarian cancer subjects with those of the control group, and in addition, to identify the 
proteins that differentiated the ovarian cancer patients from the healthy subjects.

\section{MATERIALS AND METHODS Study group}

The total study group comprised eleven patients with a median age of 57 (range 35-76) admitted to the Gynecologic Oncology Department of Poznan University of Medical Sciences. Inclusion criteria in the cancer group: a patient with suspected ovarian cancer, prior to surgical treatment, with an open cervical os, with confirmed ovarian cancer diagnosis after surgery.The cancer group comprised seven patients with advanced ovarian cancer (5 GRADE 3 serous OC, 2 GRADE 2 mucinous OC) and one patient with a borderline tumor of the ovary. Inclusion criteria in the control group: healthy patients without any oncological disease, no changes in ultrasound in the uterus and ovaries. The control group comprised three healthy patients who had been admitted because of urine incontinence. Informed written consent was obtained from each patient. The study was approved by the Poznan Univeristy of Medical Sciences Bioethical Commission.

\section{Sample processing}

Cervico-vaginal fluid samples were obtained from each patient by placing a sterile swab into the cervical canal for 10 seconds until saturation. The swab was removed and placed directly into a sterile tube containing $2 \mathrm{~mL}$ of $0.9 \% \mathrm{NaCl}$. The sample was then centrifuged at $3000 \mathrm{~g}$ for 10 minutes, and the supernatant was collected and stored at $-80^{\circ} \mathrm{C}$. The Bradford method was used for the determination of total protein concentration in each sample that we analyzed from the cancer group and the control group. The sample processing for MS/MS analysis was strictly correlated with the data received from the Bradford method analyses, to obtain a constant concentration of proteins $(20 \mu \mathrm{g} / \mathrm{mL})$ for all the vaginal fluid samples we analyzed. Thereafter $10 \mathrm{~mL}$ of each vaginal fluid sample was transferred to a $0.5 \mathrm{~mL}$ Eppendorf tube, and $15 \mu \mathrm{L}$ of ammonium bicarbonate solution $(50 \mathrm{mM})$ plus $1.5 \mu \mathrm{L}$ of DDT $(100 \mathrm{mM})$ were added. The mixture was incubated for 5 minutes at $95^{\circ} \mathrm{C}$. After cooling to room temperature, we added $3 \mu \mathrm{L}$ of iodoacetamide $(100 \mathrm{mM})$ to the reaction mixture and incubated in the dark for 20 minutes at room temperature. After this incubation, we added $3 \mu \mathrm{L}$ of trypsin enzyme solution $(0.1 \mathrm{mg} / \mathrm{mL})$ to the protein mixture and incubated for 16 hours at $37^{\circ} \mathrm{C}$. The digestion process was arrested by adding $1 \mu \mathrm{L}$ of $10 \%$ trifluoracetic acid (TFA) to the digestion mixture. Afterwards, samples were frozen and stored at $-81^{\circ} \mathrm{C}$ until the MS and MS/MS analysis.

\section{Protein identification by MALDI-TOF/TOF- mass spectrometry}

Our study used the AnchorChip Standard $(800 \mathrm{~mm}$, Bruker, Germany) target plate. As a mass standard in these experiments, the Peptide Calibration Standard II (Bruker, Germany) was used. The defined masses of calibrates allowed for the proper calibration of the apparatus and covered the mass range of 700-3500 Da. Each of the vaginal fluid samples from both the cancer patients and the control subjects was separated and fractionated using the reversed phase nano-liquid chromatography technique (Easy nanoLC, Bruker, Germany). The nanoLC apparatus was equipped with: pre-column (C18, $5 \mu \mathrm{m}, 120 \AA$, L $=20 \mathrm{~mm}$, NS-MP 10 BioSphere) and bioanalytical nano chromatographic column $(C 18,75 \mathrm{~mm} \times 15 \mathrm{~cm}$, $3 \mu \mathrm{m}, 100 \AA$ ) (Acclaim PepMap, Thermo Scientific). The following mobile phases were used: A) $0.05 \%$ TFA in water and B) $0.05 \%$ TFA in $90 \%$ acetonitrile. Analytes were eluted from the analytical column at $300 \mathrm{~nL} / \mathrm{min}$ flow during a 96-minute linear gradient from $2 \%$ to $50 \%$ of the mobile phase $B$. The eluent was mixed with HCCA (a-cyano-4-hydroxycinnamic acid) matrix and spotted onto AnchorChip standard plates ( $800 \mathrm{~mm}$, Bruker, Germany). The 384 fractions with a 15-second deposition were automatically collected on the target plates using the PROTEINEER apparatus (Bruker, Germany).

The mass spectrometry experiments were performed with the MALDI-TOF/TOF apparatus (UltrafleXtreme, Bruker), equipped with FlexControl and FlexAnalysis modules, that allowed for data acquisition and data/spectra analysis. The MS spectra were acquired in the mass range of 700-3500 Da and analyzed using the MALDI-TOF/TOF mass spectrometry instrument (Bruker, UltrafeXtreme, Germany) using a fixed laser intensity and 2500 shots per spectrum.

We used the Peptide Calibration Standard II (Bruker, Germany) as the mass standard in these experiments. Prior to each MS-analysis, the apparatus was calibrated according to the reference masses that included:bradykinin 1-7 (m/z757.3992 Da), angiotensin I and II (1046.5418 and $1296.6848 \mathrm{Da}$, respectively), substance $P(1347.7354 \mathrm{Da})$, bombesin (1619.8223 Da), renin substrate (1758.9326 Da), ACTH clip1-17 and ACTH clip 18-39 (2093.0862 and 2465.1983 Da, respectively), and somatostatin 28 (3147.4710 Da).

Based on the list of masses obtained during the MS experiments, the MS/MS mode was applied. Protein identification was performed with the ProteinScape and Mascot platform using the SwissProt database, and results with less than $1 \%$ FDR (false discovery rate) were taken into consideration. The following protein modifications were taken into the consideration: carbamidomethyl, oxidation, acetyl (N)-term and Glu-> pyro-Glu (N-term E). Protein identification search parameters were set as follows: peptide tolerance $50 \mathrm{ppm}$ and peptide charge +1 ; and up to 1 missed cleavage was permitted.

\section{RESULTS}

Our study was designed to focus on protein profile identification in cervico-vaginal fluid taken from ovarian cancer 
patients in comparison with samples from healthy control group subjects.

Using MALDI-TOF/TOF mass spectrometry (MS/MS) experiments we identified 404 different proteins in the cancer CVF samples and a further 417 different proteins in the CVF samples from the control group. At the very beginning of the study, all the proteins that we identified were divided into three separate classes: those common to both the cancer and control groups, those unique to the cancer group, and those unique to the control group.

Detailed comparison of the proteins identified in both the cancer and control group samples enabled the selection of 239 different proteins that were common to both the cancer and the control samples; 178 different proteins that were unique to the control group, and 165 different proteins that were unique to the cervico-vaginal fluid samples derived from the cancer patients. Moreover, we used the Panther Classification System program to analyze the proteins groups according to their molecular functions, biological processes, cellular components, protein class and pathways [5]. Those proteins that were characteristic of cancer were identified (Tab. 1) and those found in at least 4 (50\%) of the samples were identified (Tab. 2) and all these were analyzed according to their molecular functions, biological processes, cellular components, protein class and biological pathways. (Fig. 1). However, we particularly focused our attention on three different proteins: cysteine-rich secretory protein 3, fibronectin, and Ly6/PLAUR domain-containing protein 3 , due to their presence in 5 of the 8 ovarian cancer samples we analyzed and because of their particular biological properties.

\section{DISCUSSION}

Human cervico-vaginal fluid (CVF) is a mixture of fluids originating from the vagina, cervix, endometrium and oviduct [6]. Its composition is influenced by many factors, both hormonal changes during the menstrual cycle, as well as pathogens present in the reproductive tract [4]. Cervico-vaginal fluid can be considered as a potential source of genital tract biomarkers. The secretion is readily available [7]. For several years, interest in vaginal and cervical secretions, as potential sources of ovarian cancer markers, has been increasing. In 1978, there were cases presented that showed that the presence of cancer cells in cytology cervical smears was caused by advanced ovarian and fallopian cancer [8]. Cytological changes caused by ovarian cancer have even occurred in $19.3 \%$ of patients in the absence of changes in the cervix and endometrium. The percentage of positive smears of lavage fluid from the uterine cavity was even higher $[9,10]$. Positive cytology results were usually found in the serous type of advanced ovarian cancer (HGSC) coexisting with ascites [11]. Microscopic assessment of CVF is
Table 1. Classification of proteins identified as unique to the cance group due their molecular functions, biological processes, cellular components, protein class and biological pathways

\section{Molecular Function}

Binding (GO:0005488)

Receptor activity (GO:0004872)

Structural molecule activity (GO:0005198)

Signal transducer activity (GO:0004871) 3

Catalytic activity (GO:0003824)

Transporter activity (GO:0005215)

Biological process

\begin{tabular}{|l|l|}
\hline Cellular component organization or biogenesis (GO:0071840) & 17
\end{tabular}

Cellular process (GO:0009987)

\begin{tabular}{|l|l|}
\hline Localization (GO:0051179) & 16 \\
\hline
\end{tabular}

\begin{tabular}{|l|l|}
\hline Biological regulation (GO:0065007) & 18 \\
\hline
\end{tabular}

\begin{tabular}{|l|l|}
\hline Response to stimulus (GO:0050896) & 18 \\
\hline
\end{tabular}

\begin{tabular}{|l|l|}
\hline Developmental process (GO:0032502) & 16 \\
\hline
\end{tabular}

\begin{tabular}{|l|l|}
\hline Multicellular organismal process (GO:0032501) & 14 \\
\hline
\end{tabular}

Biological adhesion (GO:0022610)

\begin{tabular}{l|l}
\hline Locomotion (GO:0040011) & 1
\end{tabular}

Metabolic process (GO:0008152)

\begin{tabular}{|l|l|}
\hline Immune system process (GO:0002376) & 7
\end{tabular}

Cellular component

Synapse (GO:0045202)

Cell junction (GO:0030054)

\begin{tabular}{|l|l|}
\hline Membrane (GO:0016020) & 18 \\
\hline
\end{tabular}

\begin{tabular}{|l|l|}
\hline Macromolecular complex (GO:0032991) & 7 \\
\hline
\end{tabular}

Extracellular matrix (GO:0031012)

Cell part (GO:0044464)

\begin{tabular}{|l|l|} 
Organelle (GO:0043226) & 29
\end{tabular}

Extracellular region (GO:0005576)

Protein class

\begin{tabular}{|l|l|}
\hline Transporter (PC00227) & 3 \\
\hline
\end{tabular}

\begin{tabular}{ll|l|} 
Transmembrane receptor regulatory/adaptor protein (PC00226) & 1
\end{tabular}

\begin{tabular}{|l|l|} 
Membrane traffic protein (PC00150) & 1
\end{tabular}

\begin{tabular}{|l|l|}
\hline Hydrolase (PC00121) & 13 \\
\hline
\end{tabular}

Oxidoreductase (PC00176)

\begin{tabular}{|l|l|} 
Cell adhesion molecule (PC00069) & 4
\end{tabular}

Cell junction protein (PC00070)

\begin{tabular}{|l|l|}
\hline Enzyme modulator (PC00095) & 13 \\
\hline
\end{tabular}

Transfer/carrier protein (PC00219)

\begin{tabular}{|l|l|}
\hline Transferase (PC00220) & 2 \\
\hline
\end{tabular}

Transcription factor (PC00218)

Nucleic acid binding (PC00171)

\begin{tabular}{|l|l|}
\hline Defense/immunity protein (PC00090) & 4 \\
\hline
\end{tabular}

\begin{tabular}{|l|l|}
\hline Calcium-binding protein (PC00060) & 4 \\
\hline
\end{tabular}

Cytoskeletal protein (PC00085)

\begin{tabular}{|l|l|} 
Signaling molecule (PC00207) & 10
\end{tabular} 
Table 1. cont. Classification of proteins identified as unique to the cancer group due their molecular functions, biological processes, cellular components, protein class and biological pathways

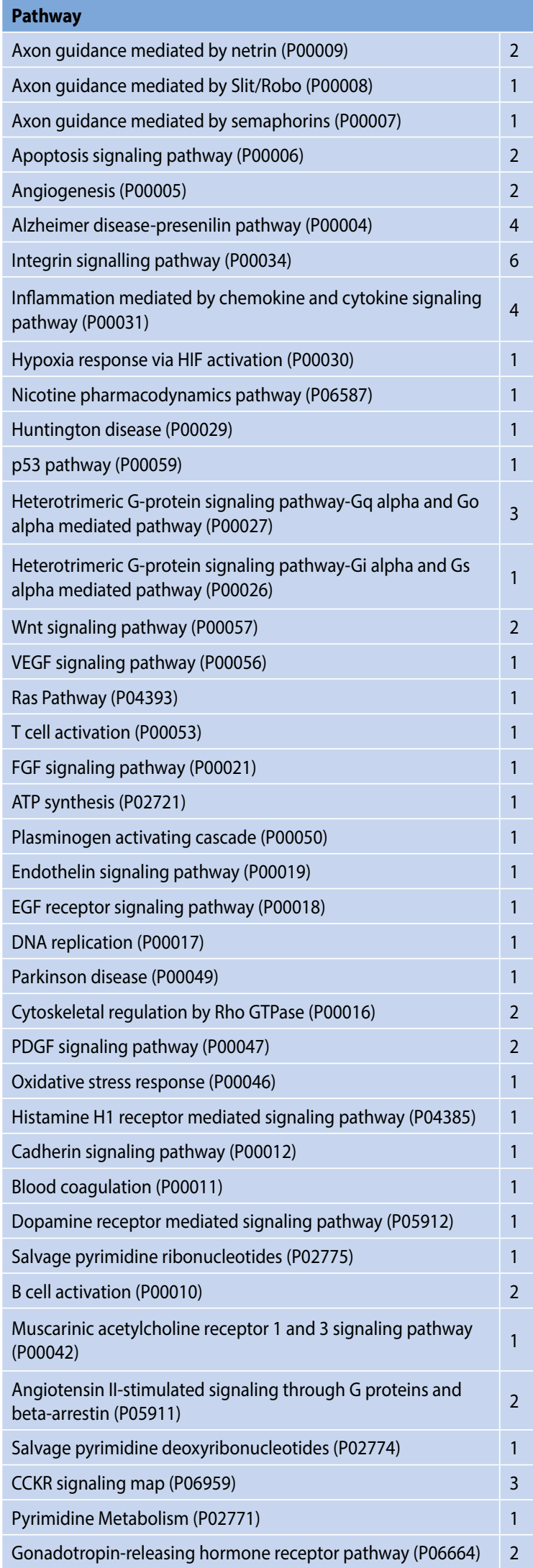

Table 2. List of proteins that differentiate the ovarian cancer group (serous ovarian cancer, mucinous ovarian cancer and borderline tumor) and control group

\begin{tabular}{|c|c|c|c|}
\hline Protein Diagnosis & $\begin{array}{l}\text { SOC } \\
(n=5)\end{array}$ & $\begin{array}{l}\text { MOC } \\
(n=2)\end{array}$ & $\begin{array}{l}\text { BT } \\
(n=1)\end{array}$ \\
\hline Alpha-2-macroglobulin-like protein 1 & 3 & & \\
\hline Serpin B-13 & 4 & & \\
\hline Acyl-CoA-binding protein & 4 & & \\
\hline Calmodulin-like protein 5 & 3 & & \\
\hline Cytidine deaminase & 2 & 1 & \\
\hline Cysteine-rich secretory protein 3 & 4 & 1 & \\
\hline Fibronectin & 2 & 2 & 1 \\
\hline $\begin{array}{l}\text { Interleukin-1 receptor antagonist } \\
\text { protein }\end{array}$ & 4 & & \\
\hline Ly6/PLAUR domain-containing protein 3 & 5 & & \\
\hline
\end{tabular}

an imprecise method in comparison with analyses of protein composition [12]. Only a limited number of proteomic studies using mass-spectrometry have been performed on cervico-vaginal fluid [7]. Analysis of CVF proteins was used in the identification of biomarkers for premature delivery, premature rupture of membranes, bacterial vaginosis and even cervical cancer $[4,13]$. Our study is the first proteomic analysis of cervico-vaginal fluid in ovarian cancer patients known to the authors, which has allowed the detection of new potential markers, that may be useful for the early diagnosis and progress monitoring of ovarian cancer.

For our preliminary proteomic study of ovarian cancer, we used MALDI-TOF/TOF MS/MS mass spectrometry coupled with the nanoLC technique for sample analysis. MALDI-TOF/TOF MS/MS is very fast and sensitive for proteomics analysis and it allows for a high throughput of protein identification [14]. However, it must be emphasized, that the presence of proteins identified by MALDI-TOF/TOF needs to be confirmed by other bioanalytical methods (e.g. immuno-assay methods).

The results of our study showed three proteins that can potentially differentiate patients with ovarian cancer from healthy patients: fibronectin, cysteine-rich secretory protein 3 and Ly6/PLAUR domain-containing protein 3. In ovarian cancer (OC), fibronectin has a well-established role in cancer metastasis. It has antiapoptotic functions, and promotes angiogenesis and the adhesion of $\mathrm{OC}$ cells to the peritoneal surface [15].

Cysteine-rich secretory protein3 (CRISP3) is involved in defense and immunity processes [16]. The expression of CRISP3 has been shown to be highly up-regulated in prostate cancer. It is associated with poor prognoses due to its role in cell invasion. Cysteine-rich secretory proteins are 


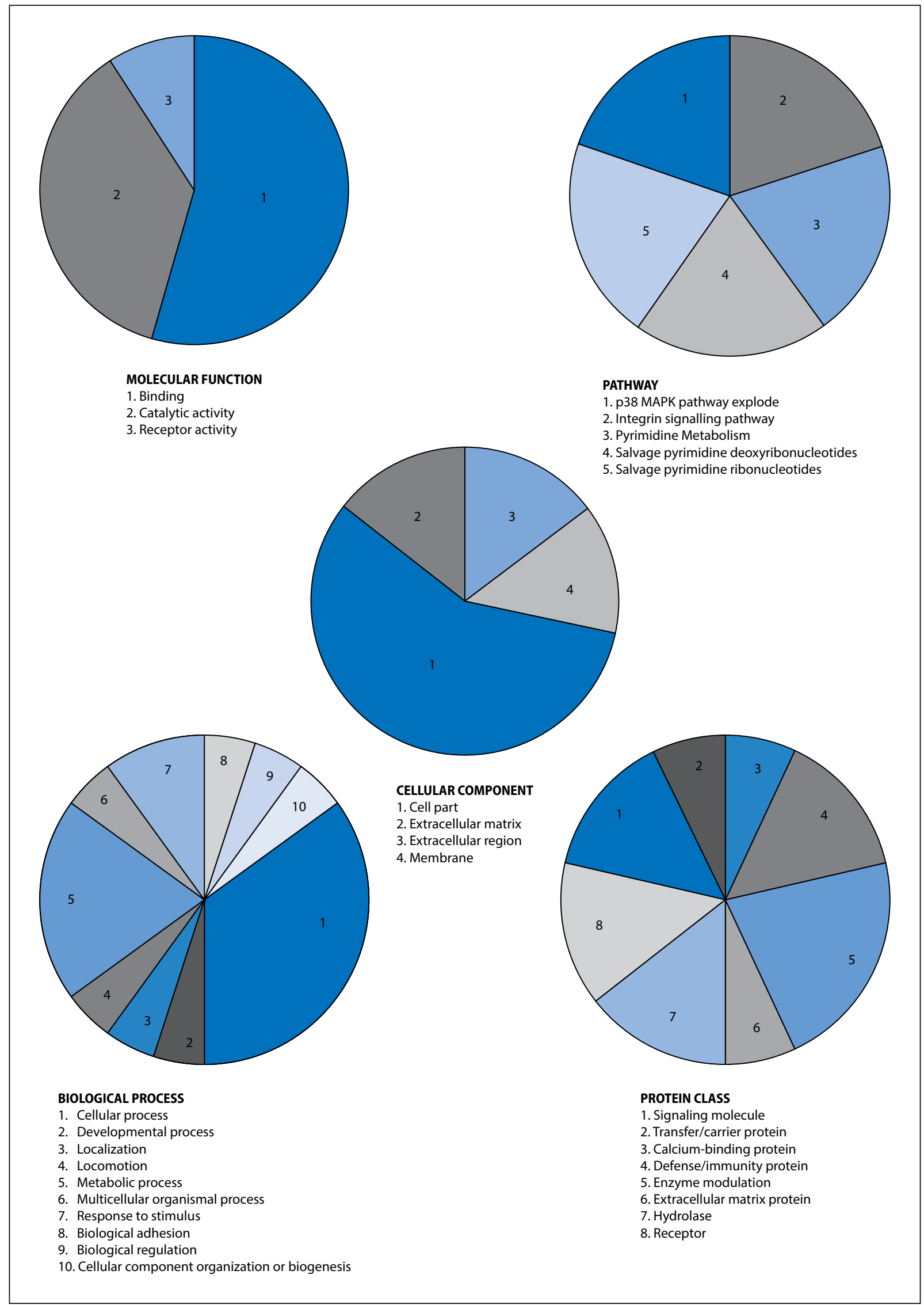

Figure 1. Graphic presentation of the functional classification (molecular functions, biological processes, cellular components, protein class and biological pathways) of all the proteins selected as characteristic of ovarian cancer patients gathered and presented at Table 1 
strongly expressed in lung adenocarcinoma tissues where they promote the migration and invasion of carcinoma cells [17]. Also high expression of CRISP3 is found in ovarian cancer tissue. However, its role and importance in this disease is unknown $[18,19]$.

Ly6/PLAUR domain-containing protein3 (LYPD3) has been identified in non-small cell lung cancer, colorectal and breast cancer. It seems to be involved in cell migration, invasion and tumor progression [20-22]. LYPD3 protein deserves special attention because it occurred in all the serous $O C$ samples in our study. It can be a marker of the most lethal serous type of ovarian cancer and enables recognition of this disease at all stages.

The proteins selected in our pilot study seem to be of great interest and to be potentially valuable markers for early ovarian cancer diagnosis. They may help in screening patients with BRCA mutation and differentiate healthy from ovarian cancer patients. However, further investigations will be necessary to prove their suitability and value as non-invasive biomarkers. It is strongly recommended that these possibilities are tested in large population studies so as to determine the levels of these proteins in vaginal fluid samples derived from ovarian cancer patients at various tumor progression stages when compared with control samples.

\section{CONCLUSIONS}

There is a characteristic proteomic pattern in the cervico-vaginal fluid of patients with ovarian carcinoma, which may allow the differentiation of cancer patients from healthy patients. Our findings indicated 3 proteins in the cervico-vaginal fluid of ovarian cancer patients: fibronectin, cysteine-rich secretory protein 3 and Ly6/PLAUR domain-containing protein3. These proteins show a strong potential to be used in multi-marker tests for the screening and detection of ovarian cancer. Our findings provide the basis for further research aimed at the early diagnosis of ovarian cancer and the reduction of mortality due to this disease.

\section{Conflicts of interest}

The authors declare no conflicts of interest.

\section{Funding}

This work was supported by Poznan University of Medical Sciences Institutional Grant No. 50201111014000257.

\section{Author contributions}

We confirm that all co-authors have been included and have contributed to the final manuscript and have approved it. EG designed the study, collected samples, analyzed the data, wrote the manuscript and prepared figures for this manuscript. JL collected samples. BU performed the
MALDI-TOF-TOF analysis, prepared figures and reviewed the manuscript. ENM provided the biological material for analysis and critically reviewed the manuscript. JM provided critical review of the manuscript.

\section{REFERENCES:}

1. Gilbert L, Basso O, Sampalis J, et al. DOvE Study Group. Assessment of symptomatic women for early diagnosis of ovarian cancer: results from the prospective DOvE pilot project. Lancet Oncol. 2012; 13(3): 285-291, doi: 10.1016/S1470-2045(11)70333-3, indexed in Pubmed: 22257524.

2. Henderson JT, Webber EM, Sawaya GF. Screening for Ovarian Cancer: Updated Evidence Report and Systematic Review for the US Preventive Services Task Force. JAMA. 2018; 319(6): 595-606, doi: 10.1001/jama.2017.21421, indexed in Pubmed: 29450530.

3. Buys SS, Partridge E, Black A, et al. PLCO Project Team. Effect of screening on ovarian cancer mortality: the Prostate, Lung, Colorectal and Ovarian (PLCO) Cancer Screening Randomized Controlled Trial. JAMA. 2011; 305(22): 2295-2303, doi: 10.1001/jama.2011.766, indexed in Pubmed: 21642681.

4. Di Quinzio MKW, Oliva K, Holdsworth SJ, et al. Proteomic analysis and characterisation of human cervico-vaginal fluid proteins. Aust N Z J Obstet Gynaecol. 2007; 47(1): 9-15, doi: 10.1111/j.1479-828X.2006.00671 $x$, indexed in Pubmed: 17261093.

5. The Panther Classification System program http://www.pantherdb.org/.

6. Michaels JEA, Dasari S, Pereira L, et al. Comprehensive proteomic analysis of the human amniotic fluid proteome: gestational age-dependent changes. J Proteome Res. 2007; 6(4): 1277-1285, doi: 10.1021/pr060543t, indexed in Pubmed: 17373841.

7. Shaw JLV, Smith CR, Diamandis EP. Proteomic analysis of human cervico-vaginal fluid. J Proteome Res. 2007; 6(7): 2859-2865, doi: 10.1021/pr0701658, indexed in Pubmed: 17567164.

8. Fox $\mathrm{CH}$. Adnexal malignancy detected by cervical cytology. Am J Obstet Gynecol. 1978; 132(2): 148-150, indexed in Pubmed: 686103.

9. Takashina $T$, Ono $M$, Kanda $Y$, et al. Cervicovaginal and endometrial cytology in ovarian cancer. Acta Cytol. 1988; 32(2): 159-162, indexed in Pubmed: 3348057.

10. Nwanodi O, Choi C, Khulpateea N. Cervicovaginal cytology and diagnosis of ovarian or peritoneal cancer: case report and literature review. Arch Gynecol Obstet. 2008; 277(2): 171-174, doi: 10.1007/s00404-007-0428-z, indexed in Pubmed: 17653735.

11. Suzuki M, Suzuki T, Matsuura M, et al. Prediction of histologic type and lymph node metastasis for advanced ovarian cancer on uterine cervical and endometrial cytology. Acta Cytol. 2010; 54(4): 575-581, doi: 10.1159/000325180, indexed in Pubmed: 20715659.

12. Otsuka I, Kameda S, Hoshi K. Early detection of ovarian and fallopian tube cancer by examination of cytological samples from the endometrial cavity. Br J Cancer. 2013; 109(3): 603-609, doi: 10.1038/bjc.2013.402, indexed in Pubmed: 23868002.

13. Zegels G, Van Raemdonck GAa, Tjalma WAa, et al. Use of cervicovaginal fluid for the identification of biomarkers for pathologies of the female genital tract. Proteome Sci. 2010; 8: 63, doi: 10.1186/1477-5956-8-63, indexed in Pubmed: 21143851.

14. Suckau D, Resemann A, Schuerenberg M, et al. A novel MALDI LIFT-TOF/TOF mass spectrometer for proteomics. Anal Bioanal Chem. 2003; 376(7): 952-965, doi: 10.1007/s00216-003-2057-0, indexed in Pubmed: 12830354

15. Kenny HA, Chiang CY, White EA, et al. Mesothelial cells promote early ovarian cancer metastasis through fibronectin secretion. J Clin Invest. 2014; 124(10): 4614-4628, doi: 10.1172/JCI74778, indexed in Pubmed: 25202979.

16. Henriksen $R$, Lundwall $\AA$, Udby $L$, et al. The expression of $\beta$-microseminoprotein but not CRISP3 is reduced in ovarian cancer and correlates to survival. Anticancer Res. 2012; 32(9): 3993-3999, indexed in Pubmed: 22993349.

17. Gong WJ, Liu JY, Yin JY, et al. Resistin facilitates metastasis of lung adenocarcinoma through the TLR4/Src/EGFR/PI3K/NF-KB pathway. Cancer Sci. 2018; 109(8): 2391-2400, doi: 10.1111/cas.13704, indexed in Pubmed: 29927028.

18. Pathak BR, Breed AA, Apte $S$, et al. Cysteine-rich secretory protein 3 plays a role in prostate cancer cell invasion and affects expression of PSA and ANXA1. Mol Cell Biochem. 2016; 411(1-2): 11-21, doi: 10.1007/s11010015-2564-2, indexed in Pubmed: 26369530. 
19. Noh BJ, Sung JY, Kim YW, et al. Prognostic value of ERG, PTEN, CRISP3 and SPINK1 in predicting biochemical recurrence in prostate cancer. Oncol Lett. 2016; 11(6): 3621-3630, doi: 10.3892/ol.2016.4459, indexed in Pubmed: 27284364.

20. Wang L, Hirohashi Y, Ogawa T, et al. LY6/PLAUR domain containing 3 has a role in the maintenance of colorectal cancer stem-like cells. Biochem Biophys Res Commun. 2017; 486(2): 232-238, doi: 10.1016/j. bbrc.2017.02.112, indexed in Pubmed: 28238780.
21. Cohen AS, Khalil FK, Welsh EA, et al. Cell-surface marker discovery for lung cancer. Oncotarget. 2017; 8(69): 113373-113402, doi: 10.18632/oncotarget.23009, indexed in Pubmed: 29371917.

22. Willuda J, Linden $L$, Lerchen $H G$, et al. Preclinical Antitumor Efficacy of BAY 1129980-a Novel Auristatin-Based Anti-C4.4A (LYPD3) Antibody-Drug Conjugate for the Treatment of Non-Small Cell Lung Cancer. Mol Cancer Ther. 2017; 16(5): 893-904, doi: 10.1158/1535-7163. MCT-16-0474, indexed in Pubmed: 28292941. 